\title{
Search Strategies Improve With Practice, but Not With Time Pressure or Financial Incentives
}

\author{
Anna Nowakowska ${ }^{1}$, Alasdair D. F. Clarke ${ }^{1,2}$, Jacqueline von Seth ${ }^{1}$, and Amelia R. Hunt ${ }^{1}$ \\ ${ }^{1}$ School of Psychology, University of Aberdeen \\ ${ }^{2}$ Department of Psychology, University of Essex
}

\begin{abstract}
When searching for an object, do we minimize the number of eye movements we need to make? Under most circumstances, the cost of saccadic parsimony likely outweighs the benefit, given the cost is extensive computation and the benefit is a few hundred milliseconds of time saved. Previous research has measured the proportion of eye movements directed to locations where the target would have been visible in the periphery as a way of quantifying the proportion of superfluous fixations. A surprisingly large range of individual differences has emerged from these studies, suggesting some people are highly efficient and others much less so. Our question in the current study is whether these individual differences can be explained by differences in motivation. In two experiments, we demonstrate that neither time pressure nor financial incentive led to improvements of visual search strategies; the majority of participants continued to make many superfluous fixations in both experiments. The wide range of individual differences in efficiency observed previously was replicated here. We observed small but consistent improvements in strategy over the course of the experiment (regardless of reward or time pressure) suggesting practice, not motivation, makes participants more efficient.
\end{abstract}

\section{Public Significance Statement}

In this study, neither time pressure nor financial incentive led to improvements in visual search strategies, ruling out motivation as the explanation for the large individual differences in search efficiency seen in previous studies. Small but consistent improvements in strategy over time suggest experience, rather than differences in motivation, could help explain why some participants are more efficient than others.

Keywords: visual search, optimality, reward, deadline, individual differences

Supplemental materials: https://doi.org/10.1037/xhp0000912.supp

We select and process only a small subset of available information. Selective sampling is achieved in part by directing the eyes to a given location, thus allowing detailed processing by the high-resolution fovea. Understanding the processes that govern eye movements can therefore help us understand and predict which aspects of the visual environment humans will be most likely to process in greater depth. In the current study, we measure the extent to which eye movements are directed to locations where central vision is most needed; that is, can they be considered efficient in how they sample information? This is not a new question: We already know that people, in general, do not tend to search very efficiently. Profound failures to find and use information effectively have been

Anna Nowakowska (1D) https://orcid.org/0000-0002-0787-4726

This work was supported by the Economic and Social Research Council [Grant (ES/S016120/1)].

Correspondence concerning this article should be addressed to Anna Nowakowska, School of Psychology, University of Aberdeen, William Guild Building, Room T32, King's College, Aberdeen, AB24 3FX, United Kingdom.Email: a.nowakowska@abdn.ac.uk shown in a range of different search contexts (e.g., Araujo et al., 2001; Clarke \& Hunt, 2016; Hout et al., 2017; Morvan \& Maloney, 2012; Rajsic et al., 2015, 2017). The question we address here is whether people can be efficient when they need to be. Specifically, we ask whether differences in priorities and motivation might explain why some individuals are inefficient with their eye movements, while others are close to optimal (Nowakowska et al., 2017).

Search for a specified target among distractors is a widely used task for studying how the visual features of the target, distractors and background influence visual selection (e.g., Treisman \& Gelade, 1980; Wolfe, 1994). But visual search entails a complex cascade of perceptual and cognitive processes, and performance is influenced not only by visual factors, but also by decision-level factors like strategy, heuristics, and biases (e.g., Clarke et al., 2019; Leber \& Irons, 2019). In the decision literature, the role of motivation, reward, effort, and tolerance for error have historically been central to methods and theories (Dreher \& Tremblay, 2009). In visual search, decision-related issues such as target prevalence (Wolfe \& Van Wert, 2010) and stopping rules (Chun \& Wolfe, 
1996) have also been studied but have been given far less attention than factors of perception and attention (Nakayama \& Martini, 2011). Accounting for contributions to variance in search performance at all stages of processing-from low-level visual properties like salience to cognitive factors like strategy and risk toleranceis fundamental to a complete understanding of visual search.

Large individual differences have been documented across a range of different visual search tasks (Irons \& Leber, 2016; Kristjánsson et al., 2014; Nowakowska et al., 2017). "Individual differences" in this context is used in the literal sense that individual participants do not converge on a single pattern but display a wide range of different behaviors under the same conditions. A concerted effort to try and account for these differences has had mixed results. Kristjánsson et al. (2014) showed individual differences in search strategy were apparent during complex conjunction-based foraging but not during easy feature-based foraging. They suggested the cognitive capacity of individual foragers might explain the differences. Similar between-subjects variation was documented in oculomotor orienting (Tagu \& Kristjánsson, 2020), with a suggestion that selection modality (using a mouse, touch screen or eye movements) mediated individual differences. Although these differences appear to be relatively stable over time within an individual, a given person's performance on one search task appears to tell us very little about how they will perform on the others (Clarke et al., 2020), and efforts to explain the differences in search using other psychometric tests have not, to date, been very successful (e.g., Irons \& Leber, 2018; Jóhannesson et al., 2017).

Persistent individual differences present challenges for efforts to develop models of visual search or even to draw simple conclusions about how people, as a group, search for objects. Human search has been proposed to be consistent with an "ideal" model, in which each fixation is directed to locations that maximize the accrual of new information (Najemnik \& Geisler, 2005; 2008). On the other end of the spectrum, search has also been demonstrated to be consistent with a stochastic model, in which fixations are randomly selected from a population of fixations constrained by habit and biases. Nowakowska et al. (2017) demonstrated that human fixation behavior is in fact consistent with both of these models, in that some individuals were consistent with the predictions of the ideal model and some with a stochastic model. Other individuals, however, were consistent with neither account. While these individual differences do partially resolve the contradiction between different accounts of fixation selection, they also lead to new, more complicated questions about why these differences exist and how to develop a model of search that accounts for them.

In the paradigm that uncovered these large individual differences, called split-half line search (SHLS), participants searched an array of lines for a line tilted $45^{\circ}$ to the right while their eye movements were recorded. The mean orientation of the distractors was perpendicular to the target, but on one side the distribution of distractor orientations was narrow ("homogeneous"), and on the other side the distribution was much wider ("heterogeneous", see Figure 1). When the target is present on the homogeneous side, it can be easily detected using peripheral vision. When the target is present on the heterogeneous side, foveal vision is required to discriminate it from the distractors. The SHLS experiment can therefore measure the extent to which eye movements are directed to the locations where foveal vision is required. In other words, eye movement efficiency is operationally defined as the proportion of
Figure 1

Example of the Search Stimuli

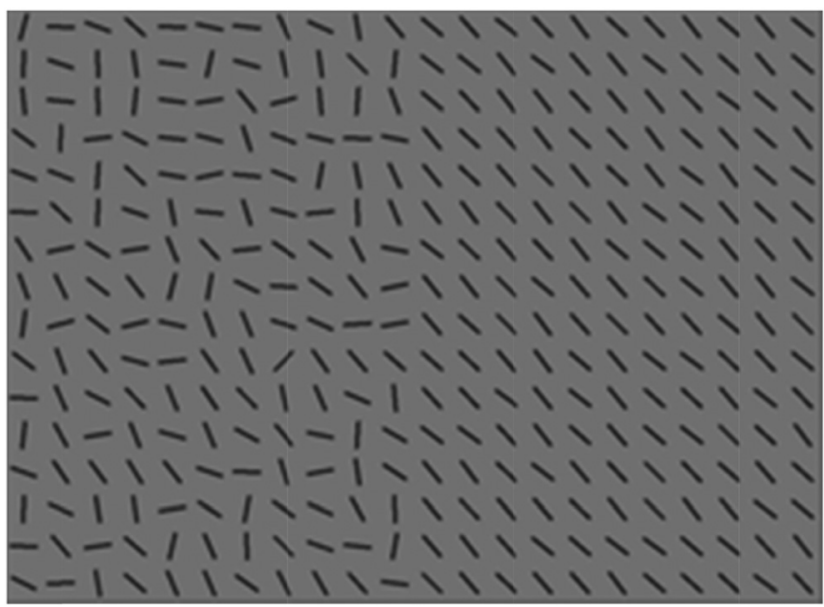

Note. The target is present on the heterogeneous side of the array (10th row, 8th column).

eye movements directed to the heterogeneous side of the search array on target absent trials ${ }^{1}$. Models of optimal visual search in which fixation selection is based on expected information gain (e.g., Najemnik \& Geisler, 2005) would predict this proportion to approach 1. Nonetheless, in Nowakowska et al. (2017), nearly half of the first five search related fixations on each trial were made to the homogenous (easy) side. Underlying this general lack of efficiency was the large variation between participants already mentioned, with some participants performing near optimal, some being extremely inefficient, and the rest falling in between. Two factors contributed to this search inefficiency: making more fixations than was necessary, and not directing eye movements to the locations that would be most informative for finding the target.

It is possible, however, that participants are capable of searching more efficiently but, for reasons of motivation or distraction, fail to implement an efficient strategy. In Nowakowska et al. (2017), even though participants were encouraged to be as fast and as accurate as possible, participants had 60 seconds to search before each trial timed out. Thus they could "afford" to make the redundant confirmatory eye movements that were observed without sacrificing accuracy. Some participants may prefer to explore every region to increase their confidence that the target is not present, while others prefer to respond as quickly as possible. Indeed, there was a very wide range of reaction times, particularly on target absent trials, on which individual median reaction times ranged from less than 2 seconds to more than 15 . If the display time had been limited, fixations on the easy side would leave no time for inspecting the hard side while not adding to the accuracy to detect targets on the easy side (as it is already at ceiling). Therefore, one factor that could induce more efficient fixation behavior could be tighter restrictions on how long participants can spend searching.

Time pressure was found previously to affect decision-making and judgment, in that observers tended to use different decision

\footnotetext{
${ }^{1}$ We acknowledge that many other definitions of "efficiency" exist, both within and beyond the visual search literature (e.g. Liesefeld \& Janczyk 2019; Townsend \& Ashby, 1983; Wolfe, 1998).
} 
rules when time was constrained (Edland, 1994; Svenson \& Edland, 1987). In consumer choice tasks, time pressure has been associated with decreased average fixation duration (Pietersa \& Warlopb, 1999) and a reduction of the amount of information fixated (van Herpen \& Trijp, 2011). Time pressure also moderated the effect of visual saliency on consumer choices (Mormann et al., 2012). Specifically, the visual saliency of an item was a better predictor of consumer choices than their personal preferences when the time to make a choice between a number of items was constrained. This study in particular suggests that time constraints lead to the "rational" decision being overridden by the low level saliency of the visual items. In the context of eye movements during search, however, we predict the opposite effect, where the time constraints would encourage more rational fixation behavior. In Thornton et al. (2019), participants adapt strategies according to task demands, suggesting participants might be able to switch to more efficient eye movement behavior when the time to complete the task is limited. With time constraints, inefficient fixations could lead to errors and trial time-outs, which may be more effective in motivating efficient fixations than search speed.

Another way to increase efficiency may be financial incentives. The study of reward as a mechanism driving visual selective attention has been of close interest to researchers in recent years (Anderson et al., 2011; MacLean \& Giesbrecht, 2015; Navalpakkam et al., 2010). Learning to associate visual stimuli with reward creates a persistent attentional bias that continues to involuntarily drive attentional selection in favor of previously rewarded stimuli, even when those stimuli are no longer task relevant or rewarded (Anderson et al., 2011, 2012; Anderson \& Yantis, 2012), and the modulating effect of reward might last up to half a year after acquisition (Anderson \& Yantis, 2013). In visual search, Zhang et al. (2017) found evidence that when searching for multiple targets, humans change their strategy in response to different patterns of reward, searching for longer when reward were high, and for less time when they were low. Navalpakkam et al. (2010) model visual search by presenting participants with brief search displays containing two targets associated with a monetary reward. The reward value and salience associated with the targets varied from trial to trial, and both measures of performance used in the experimentsaccadic eye movements and key presses-indicated that strategy was flexibly adapted to the changing demands to achieve near optimal performance, in line with assumptions of the ideal Bayesian Observer model. It is important to note, however, that optimal saccades were directly associated with a reward. In the real world, the aim of the eye movements is to accumulate "evidence" to aid the decision/action to be rewarded (Eckstein, 2011).

Chelazzi et al. (2013) outline two potential mechanisms modulating attentional selection by means of reward. First, attention could be biased through direct incentive motivation. Varying reward with respect to different spatial locations or stimuli is associated with preferential deployment of attention to the location or stimuli associated with the maximum incentive value (see e.g., Small et al., 2005). Second, the availability of the reward biases attentional priority by means of learning, which results in alteration of the current performance by stimuli previously associated with a reward (see e.g., Della Libera \& Chelazzi, 2009). In the context of eye-movements, Hayhoe and Ballard $(2005,2014)$ link the evidence from neural recording, reinforcement learning studies, and research into graphic simulations and suggest a model of eye movements integrated with the ongoing task. This model samples multiple sources of dynamic information at moments of increasing uncertainty and is shaped by reward. In order to make a decision about the relevance of a stimulus, observers need to learn through practice (Haider \& Frensch, 1999); this learning could be achieved through feedback, associated with a reward value of attending to the relevant stimuli (Hayhoe \& Rothkopf, 2011; Tatler et al., 2011).

In our first experiment, we manipulated the duration of the search display and measured the effect on eye movement efficiency. Participants were asked to complete two blocks of the split-half visual search task from Nowakowska et al. (2017). In one block, participants only had two seconds to search before the search array was masked (we will call this the brief condition); after two seconds a gray mask covered the search array, but participants could still respond. The dependent variable of interest in the experiment is eye movements; to be useful, they need to be executed while the visual display is presented. With a two-second display, more efficient eye movements become a requirement for an accurate response. We left manual response times unconstrained to avoid unnecessary pressure to press response keys quickly, which would lead to errors and data loss. In the other block, the observers had up to $60 \mathrm{sec}$ to view the search array and respond (which we will call the long condition). Half of the participants completed the brief block first, and the other half completed the long block first. Participants completed both brief and long conditions because this permits a measurement of the effect of timing within each participant, which is important given the very large individual differences in search efficiency observed in previous studies.

In Experiment 2 we manipulated incentives offered to participants in order to examine the effect of reward on search strategy. We divided participants into two groups: the reward group and the flat payment group. Both groups completed two blocks of the split-half search task, and both groups were initially informed that they would receive $£ 5$ after completing the first block. After completion of the first block, participants in the reward group were told that they would receive a $£ 5$ additional reward if they responded $10 \%$ faster than they did in the first block and a $£ 10$ additional reward if they responded $20 \%$ faster, thus potentially topping up their reimbursement to $£ 10$ or $£ 15$. We also stipulated that accuracy in the second block had to stay at least as high as accuracy in the first block to receive the additional reward. Participants in the reward group were therefore highly motivated to improve their search speed. Participants in the flat payment group were told they would receive an additional $£ 5$ after completing the second block regardless of their performance.

If individual differences observed in previous studies were due in part to differences in error tolerance or motivation to search quickly, we should see an overall increase in eye movement efficiency, as measured by a higher proportion of fixations directed to the heterogeneous side of the search array, under conditions of restricted time and/or financially incentivized search speed. We should also see an overall reduction in individual variation under these conditions, as all individuals should approach the ceiling of efficiency. Baseline conditions should replicate the wide variation in individual search efficiency seen in previous experiments (Clarke et al., 2020; Nowakowska et al., 2016, 2017, 2019). Search strategy is also likely to fluctuate within participants, not only between experimental blocks, but also within the blocks, or even within the trial. We therefore also present an exploratory 
analysis across both experiments of how search efficiency changes over blocks, trials, and fixations.

\section{Experiment 1: Deadline}

\section{Method \\ Participants}

18 naive observers gave informed consent to participate in the experiment $($ females $=9$; age range $=17-33$; mean age $=24.1$ ). The experimental protocol was reviewed and approved by the Aberdeen Psychology Ethics Committee.

\section{Stimuli and Procedure}

The search arrays were similar to the ones used in Nowakowska et al. (2017). Line segments were aligned in 22 columns and 16 rows on a uniform gray background. The line was $1.2 \mathrm{~cm}$ long $\left(1.5^{\circ}\right.$ at a viewing distance of $\left.45 \mathrm{~cm}\right)$. The target line was always tilted $45^{\circ}$ to the right. The distractors had random orientations, sampled from a uniform distribution with a mean angle perpendicular to the target angle. The target was easy to spot on one half of each search array and difficult on the other (see Figure 1 for an example). Difficulty was manipulated by varying the range of the distractor line orientations, with a narrow range (right side of Figure 1) producing easy search and a wider range (left side of Figure 1) producing more difficult search. In Nowakowska et al. (2017), the distractor orientations were sampled from a $30^{\circ}$ range of distractor line orientations ("homogeneous") or a $106^{\circ}$ range ("heterogeneous"). However, in a related study, Nowakowska et al. (2019) found that participants tended to improve at spotting the target line on the heterogeneous background with practice, independent of their eye movement strategies. To ensure heterogeneous-side targets continue to not be visible in the periphery for the entire duration of the current experiment, we made the search more difficult on the heterogeneous side by increasing the range of distractor angles to $120^{\circ}$. We also slightly decreased the range of distractor orientations on the homogeneous side to $18^{\circ}$ (thus making the line array even more uniform).

To compare the extent to which participants explored the homogeneous vs heterogeneous side of the array, all the search arrays were translated into the same visual coordinate space, with 0 at center, negative values on the heterogeneous side, and positive values on the homogenous side. We only use target absent trials in this analysis to ensure we examine "searching" fixations, rather than those directed toward found targets.

There were 96 arrays of lines in each of the two blocks (192 trials in total), and the target was present on half of the trials. Whether the heterogeneous side was on the left or right was randomly determined on each trial. The side of the target relative to the search difficulty was counterbalanced. The target could be located in any of the possible locations apart from the middle two vertical columns. Participants were told they would see an array of line segments on the screen and that their task was to determine whether a line tilted $45^{\circ}$ to the right was present among other lines. Participants were asked to respond as quickly and accurately as possible by pressing either the left (present) or right (absent) arrow key. In one block, the search array would remain on the screen until the participant responded. In the other block, the search array would remain on the screen for two seconds. Participants were told which condition they were about to complete before each block started. Participants were encouraged to be as fast and as accurate as possible in both conditions. The order of blocks was counterbalanced, with nine participants completing each order.

Each trial consisted of a black fixation point (letter $\mathrm{x}$ ) subtending $1.5 \mathrm{~cm} \times 2.5 \mathrm{~cm}\left(1.9^{\circ} \times 3.1^{\circ}\right)$, presented at the center of the computer screen. We asked participants to fixate the center of the fixation cross. On the press of a space bar the fixation cross disappeared, then the array of line segments was displayed until the participant made a response (or timed out after 60 seconds). Auditory feedback in the form of a beep immediately followed incorrect key presses. Before the start of the experiment, participants underwent a nine-point calibration sequence and a block of 10 practice trials.

A 19 in. CRT ViewSonic Graphics Series G90fB monitor with a resolution of $1024 \times 768$ and refresh rate of $100 \mathrm{~Hz}$ was used to display stimuli. MATLAB 2014 running Psychtoolbox (Brainard, 1997; Pelli, 1997) and EyelinkToolbox (Cornelissen et al., 2002) was used to present stimuli and record data on a Macintosh PowerMac. A desktop-mounted EyeLink 1000 eye tracker sampling at $1000 \mathrm{~Hz}$ was used to record the position of one eye. The right eye was recorded by default; however, if we failed to calibrate the right eye, the left eye was used instead.

\section{Analysis}

We summarize each participant's scan-path by reporting the proportion of fixations made to the heterogeneous side of the display. Following Clarke et al. (2020), we only consider fixations 2-6 made during correct target absent trials. We use only target absent trials to ensure all the fixations are related to the process of searching, rather than being target-directed. We limit to the first five search-related fixations (the first fixation was always at the center) for two reasons. First, nearly all target absent trials contain at least six fixations, so limiting to six means, we get an unbiased sample of search behavior. Second, search strategies vary most widely early in the trial; later on, participants tend to converge on the heterogeneous side, leaving little variation in this metric.

We used Bayesian generalized multilevel linear models to investigate how our experimental manipulations influenced visual search strategy. Because we are modeling proportional data, we use beta distributions, which are defined over $(0,1)$. The small number of 0 and 1 data points values are set to .01 and .99 , respectively. For both Experiments 1 and 2, the model formula, we will use the maximal random effects structure.

Models are fit using R (v3.6.1) with the brms (v2.12) package (Bürkner, 2017). In general, we follow the advice given by McElreath (2020), using weakly informative priors and plotting the predictions made by both the prior and posterior distributions. In the text, we use the $95 \%$ highest posterior density interval (HPDI) to summarize the posteriors from our models. HPDI should be interpreted in the following way: If we were to sample a point at random from the distribution, there is a $95 \%$ chance that it would be within this interval. In order to assess the extent to which the results support our hypothesis, we need 
to estimate the difference between the distributions. One of the advantages of Bayesian analysis is that this calculation is comparatively simple, and we can directly calculate the probability that the difference is greater than zero (given our data and assumptions).

\section{Power Analysis}

A simulation-based power analysis was carried out by assuming a target posterior distribution in which the average participants fixated the heterogeneous side of the display $51 \%$ of the time in the long condition and $57 \%$ of the time in the brief condition. We selected $51 \%$ based on results from previous experiments (which show a very slight tendency to fixate the heterogeneous side) and $57 \%$ as a hypothetical small increase in this tendency associated with our intervention. This is a highly conservative estimate of the difference we expect under the hypothesis; if a lack of motivation makes some participants inefficient, our intervention should produce close to $100 \%$ heterogeneous fixations. We selected this far smaller number $(57 \%)$ to ensure we have the power to detect effects that partially confirm the hypothesis. We then simulated our experiment by sampling data for 15 observers each carrying out 32 target absent trials. This simulated dataset was then analyzed as if it were the real data, and the result was summarized by $p(x>0 \mid d)$ : the probability, given the data (d), that the difference between the brief and long conditions (x) was greater than zero. Over 50 repetitions of this process, all values of $p(x>0 \mid d)$ were greater than .95 . This analysis demonstrates that our sample size $(\mathrm{n}=18)$ is sufficient to detect relatively small differences (i.e. $51 \%$ compared to $57 \%$ ) in eye movement efficiency. For the full specification of our data generation process, including all code, please see the online supplementary materials.

\section{Results}

\section{Manual Response Data}

We report search accuracy and reaction time (RT) in Figure 2. It is clear from this figure that median brief reaction times (seconds) were faster $(2.07, S D=1.00)$ than long $(4.75, S D=9.25)$; this shows that taking the information away after 2 seconds led to faster responses than displaying it (more or less) indefinitely, as would be expected. There was a modest speed-accuracy trade-off, with accuracy $(\%)$ lower in the brief $(74.30, S D=6.94)$ than the long condition $(81.82 \%, S D=7.91)$.

The results across target present and absent trials look as expected, with slower reaction times when the target is absent or when it is present on the heterogeneous side of the array relative to the homogeneous side, when it is detected very quickly. Similar to previous experiments (Nowakowska et al., 2017; Clarke et al., 2020), participants tend to report that the target is absent when they are unable to find it, so accuracy is high in target absent trials and low when the target is present on the heterogeneous side. The level of difficulty of the search task was increased slightly in the current experiments, compared to the original experiment (Nowakowska et al., 2017), because of the findings from a related experiment (Nowakowska et al., 2019) that observers' ability to spot the target using peripheral vision improves quickly, even on the heterogeneous side. One minor concern about this change was that when faced with this more difficult search task, participants might have given up searching at all. However, this concern was alleviated by the long reaction times observed when the target was present on the heterogeneous side or absent; this suggests they were continuing to try to find the target even when it was not immediately visible. Participants were also successful in finding the target

Figure 2

Accuracy (Top) and Reaction Time (Bottom) Data in the two Conditions (Long and Brief) for Each of the Target Conditions
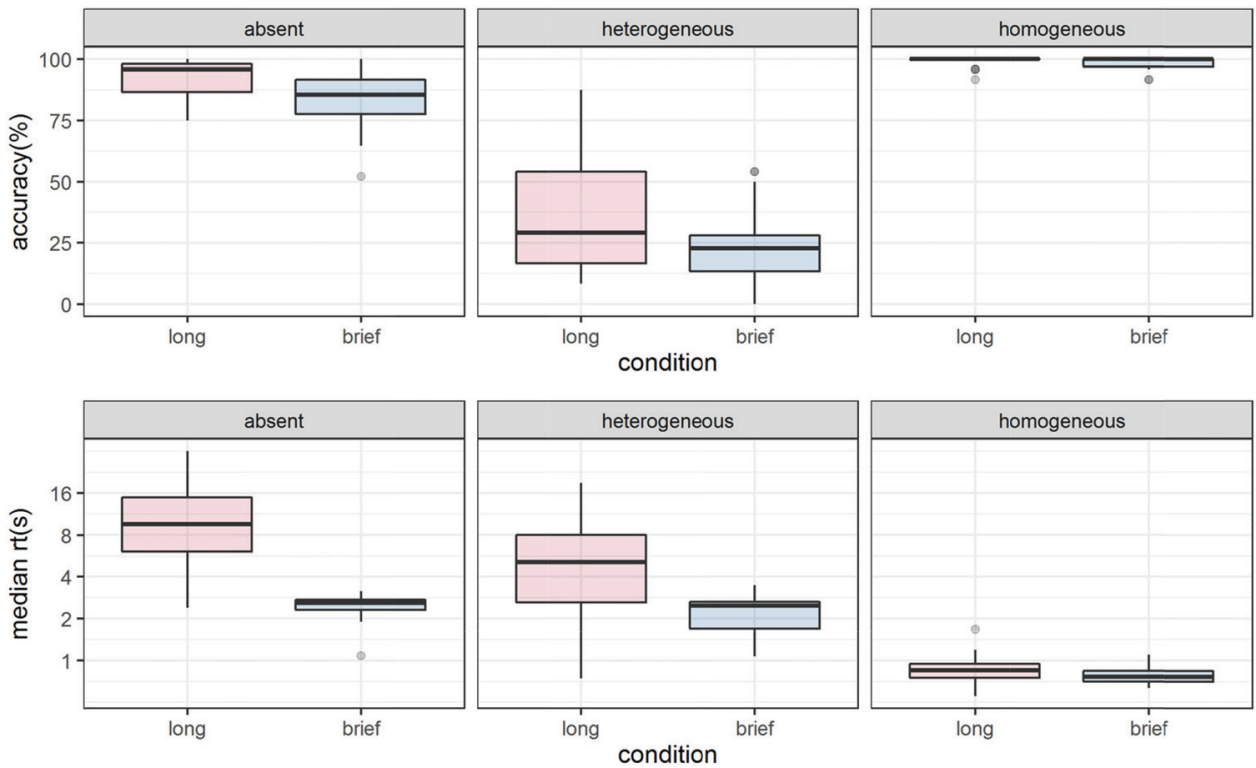

Note. The lower and upper hinges correspond to the first and third quartile. See the online article for the color version of this figure. 
Figure 3

(a) The Mean Proportion of Fixations to the Heterogeneous Side for the Long and Brief Condition for Individual Participants, (b) The Prior Predictions of Our Model, (c) The Posterior Predictions, After Fitting the Model to Our Data

(a) Empirical

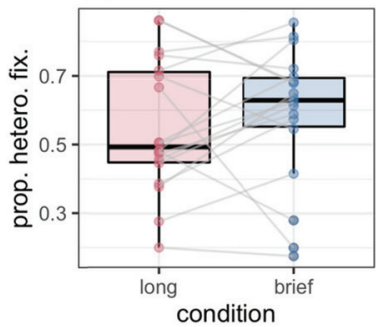

(b) Prior

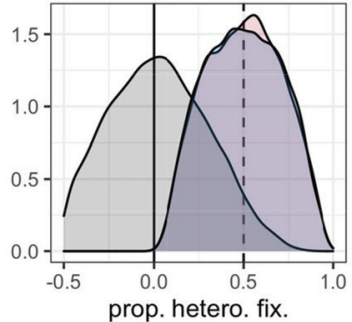

(c) Posterior

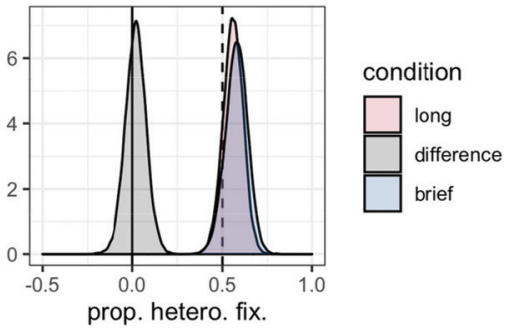

Note. In (a), the lines connect mean proportion in the two conditions for each individual participant. Both (b) and (c) show predictions for the average participant, i.e. assuming that all random effects are set to zero. See the online article for the color version of this figure.

on the heterogeneous background on around a third of the long-duration trials.

\section{Eye Movement Efficiency}

Figure 3a shows our measure of search efficiency: the proportion of the first five search-related fixations on target absent trials that were made on the heterogeneous side of the display. Similarly to the previous experiment of Nowakowska et al., 2017, a strict criteria for an optimal strategy in this experiment would be to not look to the homogeneous side at all, leading to proportions close to 1 . As we can see in Figure 3, most participants waste a considerable number of fixations on the homogeneous side, and there was large variability between participants in terms of the proportion of fixations directed to the heterogeneous side (as seen in the individual points for each participant). Despite these individual differences, the strategies appeared relatively stable, with participants implementing a similar eye movement strategy across both blocks (discussed in greater detail below).

As can be seen in Figure 3c, there is no evidence of a difference in the proportion of fixations directed to the heterogenous side between brief and long exposure conditions. The HPDIs are [.45, $.67]$ and $[.46, .69]$ for the long and brief conditions respectively. The probability that the difference between the two conditions is greater than 0 , given the data, is .62 . Therefore, we conclude that the addition of a short time limit did not substantially or consistently increase search efficiency.

\section{Experiment 2: Reward}

\section{Method \\ Participants}

42 naive observers participated in the experiment (reward: females $=18$; age range $=19-65 ;$ mean age $=24.90$ [SD = 10.12]; flat : females $=16$; age range $=19-36$; mean age $=23.15[S D=$ 4.24]). Three participants were excluded from further analysis: Two participants scored below 50\% correct in the easy condition (i.e. when the target appeared on the homogenous side), and one participant had a mean RT of over $8 \mathrm{~s}$ on these same trials, suggesting these three participants did not understand the task. Thus, we had 18 observers in the flat payment group and 21 in the reward group. The protocol was reviewed and approved by the Aberdeen Psychology Ethics Committee. Participants in the control group always received $£ 10$ for participating in the experiment; participants in the reward group received between $£ 5$ and $£ 15$ (mean was $£ 8.41$ ).

\section{Material and Procedure}

The stimuli were exactly the same as in Experiment 1 . The procedure was also the same as in experiment 1 long condition, and additional reward instructions were given to participants in the reward group following the completion of the first block. At the end of the first block of 96 trials, the experimenter told participants that they would be paid $£ 5$ for their participation in the experiment regardless of their performance. The experimenter added that the participant would receive an additional $£ 5$ if they improved their overall reaction times (decrease RTs) in the second block by at least $10 \%$. If they improved their performance by at least $20 \%$, compared to their performance in the first block, they would receive an additional $£ 10$. To receive the additional reward for improvement in RT, their accuracy had to stay at least the same as in the first block. Participants were given this information only after completing the first block to ensure they would not deliberately underperform to make receiving the maximum reward easier to achieve. Participants were not given feedback on their RT after every trial but they were given feedback at the end of block one and block two.

\section{Power Analysis}

As with Experiment 1, we ran a simulation of the experiment in which we assumed a relatively small difference between conditions and simulated data and then ran the analysis as if it were the real data. Over 50 simulations with 40 observers each completing 32 target absent trials, we found that the minimum $p(x>0 \mid d)$ to be over 95 . Please see online supplementary materials for full technical details.

\section{Results}

We preregistered the methods, hypothesis, and analysis plan for this experiment (https://osf.io/efg8n). However, the preregistered 
analysis plan specified an ANOVA, and after completing the analysis and developing an approach to the exploratory analysis that follows, we decided to apply a consistent Bayesian approach to the statistical analysis throughout the study. Both analyses lead to the same conclusions, and the original, preregistered ANOVA is presented in the online supplementary materials.

\section{Accuracy and $R T$}

From Figure 4, it is clear that there are baseline differences between the control (flat) and reward groups: Even though the conditions are identical in block 1, performance in the control group is slower. We are confident that these differences are due to chance; participants were randomly assigned and did not know what group they were in until after the end of block 1. Participants in the reward group were overall faster but less accurate (Figure 4 top row), and a few participants in the flat payment group who had extremely long reaction times inflated the RTs in that group. We therefore need to be careful to focus on the performance difference from block 1 to block 2 rather than to interpret the differences between groups in the second block alone. Together the RT and accuracy data suggest that participants improved their performance in the Block 2 compared to Block 1 both in terms of RT and accuracy, but the type of payment did not have significant influence on the performance.

\section{Eye Movement Efficiency}

As in Experiment 1, in this analysis we only include the first five search-related fixations on correct target absent trials and exclude the first (central) fixation and any subsequent fixations that fell inside of the central 64-pixel strip of the search array (approx. $1^{\circ}$ ). The proportion of these fixations falling on the heterogeneous (hard) side is shown in Figure 5a. Two participants in the reward group (second block) do not have any trials that meet this further criterion, hence we were left with 18 participants in the flat payment group and 19 participants in the reward group.

As can be seen in Figure 5c, there is some evidence that participants in both the flat and reward conditions improved their eye movement efficiency from block 1 to block 2 (HPDIs of $[.04, .13]$ and $[-.02, .12]$ respectively). However, there is no evidence that the size of this improvement was larger for participants in the reward condition: The probability that the difference between the two conditions is greater than 0 , given the data, is $p(x>0 \mid d)=$ .10. Therefore, we conclude that the introduction of reward did not lead to more efficient visual search strategies.

The correlation between the proportion of fixations to the hard side in the first and second block was relatively high $(r=.63,95 \%$ confidence interval $[.17, .83])$. The finding that the pattern is variable between participants but consistent over repeated measures is consistent with the results of Clarke et al. (2020), who found similar test-retest reliability [.63-.86] The new, lower correlation seen in the present study is likely due to the experimental manipulation between one block and the next, given that the correlation between block 1 and block 2 in the flat payment condition alone was stronger $(r=.90$, CI $[.77, .97])$.

\section{Exploratory Time Course Analysis}

In the two experiments described above, neither the time pressure nor the reward manipulation reliably improved search strategies. What did emerge were small but consistent effects of practice. The improvement from block 1 to block 2 in search efficiency in Experiment 2 (also observed in Experiment 1, as discussed below) suggests a gradual

Figure 4

Accuracy and Median Reaction Times for the Three Target Conditions in the First and Second Block for Flat and Reward Groups
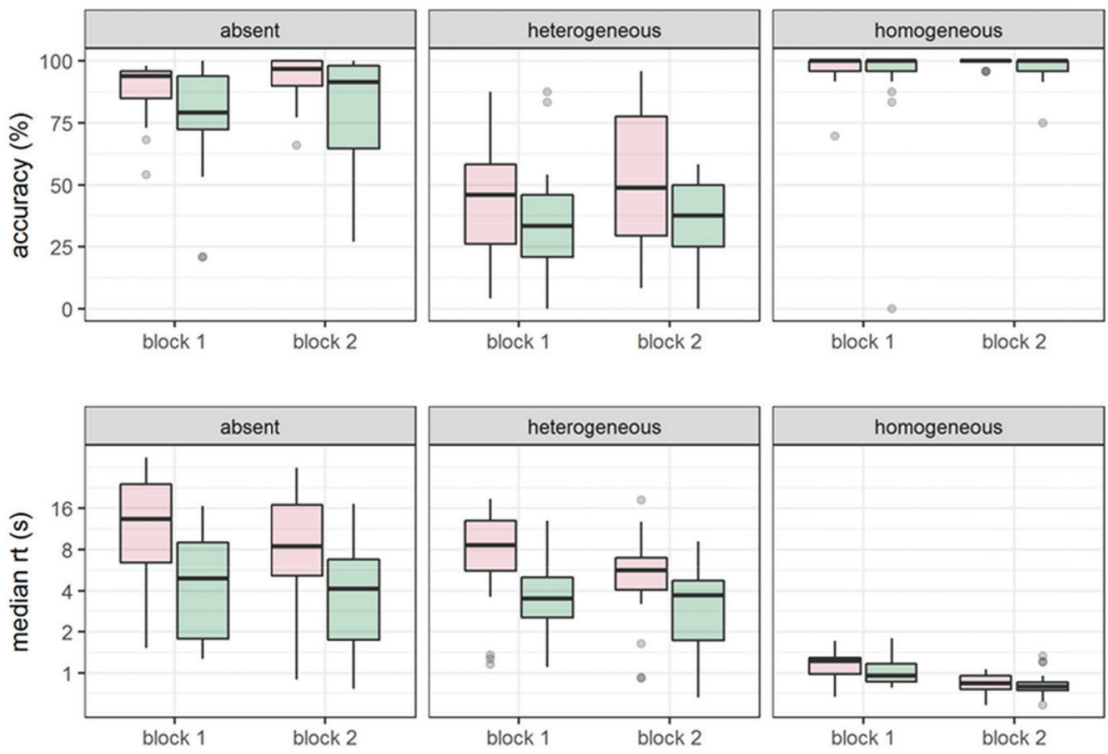

condition

宁 flat

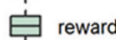

Note. Block 1 was identical for both groups, so the differences in Block 1 seen here are baseline group differences. See the online article for the color version of this figure. 
Figure 5

(a) Proportion of Fixations to Heterogeneous Side on Target Absent Trials Only, (b) The Prior Predictions of Our Model, (c) The Posterior Predictions After Fitting the Model to Our Data

(a) Empirical

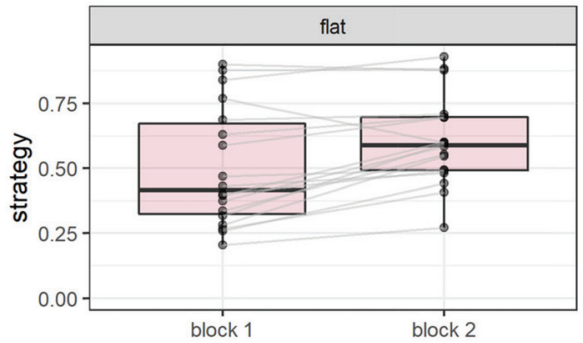

(b) Prior

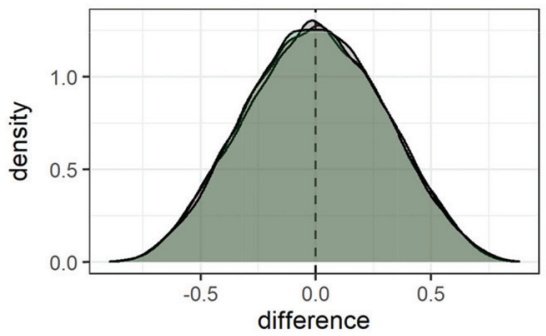

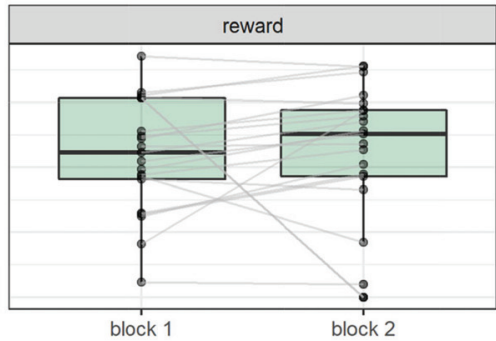

Posterior Predictions

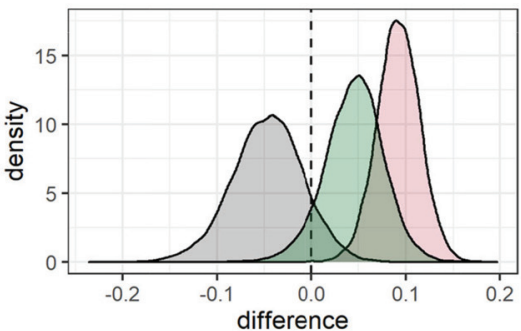

cd

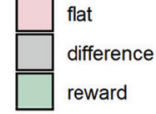

Note. (a) The left panel shows participants who received flat payment, and right panel those who were offered a reward for improving performance. Most participant improve their strategy (make more eye movements to the heterogeneous side) in the second block, regardless of the payment condition. Both (b) and (c) show predictions for the average participant, i.e. assuming that all random effects are set to zero. The $\mathrm{x}$ axis shows the difference in efficiency (Block 2 - Block 1). See the online article for the color version of this figure.

process whereby classes of eye movements associated with faster search (those directed toward the heterogeneous side) become more likely, and less efficient eye movements (those directed toward the homogeneous side) become less likely. Participants were unable to implement better strategies when they were incentivized, suggesting these improvements must instead be gradually acquired through practice.

Practice effects are important because they offer a possible explanation for the large individual differences observed between participants in this and previous experiments (Clarke et al., 2020; Nowakowska et al., 2017, 2019); perhaps these differences are, at least in part, a consequence of sampling each participant at a different stage of learning, rather than (or in addition to) being a stable indicator of cognitive style or personality (i.e. Lonnqvist et al., 2020). While some personality and cognitive characteristics (such as conscientiousness and working memory capacity) seem likely to contribute to certain types of search skills, this has proven difficult to demonstrate empirically (e.g., for a recent review see Leber \& Irons, 2019). Differences in experience with search and in learning rates for particular skills and tasks are also likely to contribute to individual differences in search, and the practice effects observed in the current experiments offer an opportunity to quantify and understand their contribution.

In this final section, we therefore combine the results from the two experiments to explore the effects of practice on search strategy. Four specific questions can be addressed in this analysis. The first relates to a possible limitation of Experiment 1, which is that we counterbalanced the conditions without regard to possible effects of the order of exposure. If restricted exposure to the search array leads to more efficient search strategies, participants who performed the brief condition first may utilize a more efficient strategy not only in the timed condition, but also in the long condition that follows it. This would have reduced the differences between conditions for this group. A second question is the rate of change over time; do participants gradually improve their strategies, or is there a step-change between blocks 1 and 2 in the experiment where a more efficient approach is discovered? Third, is the rate of learning similar across the different conditions (i.e. with time constraints or reward), or do differences emerge with a more nuanced comparison?

A fourth and final question explored in this analysis is how search strategies unfold over each trial. In this study and previous ones, we have restricted our analysis of search strategy to the first six fixations on target absent trials in part because these are the most diagnostic; by the end of each trial all participants tend to direct the majority of their fixations to the heterogeneous side by necessity. What differs between participants is the earlier stages of the trial, in which some participants direct fixations to the heterogeneous side from the start, and others seem to check the homogeneous side first, or make more distributed eye movements, before focusing on the locations that yield new information. In the analysis below, we look at practice effects separately for each of the first six fixations on each trial to better understand whether practice effects are making all fixations uniformly more efficient or if 
they are improving earlier or later fixations exclusively. These analyses are exploratory: Our goal is to describe the pattern of practice effects and quantify their size and stability, not to test any particular hypothesis.

\section{Method}

\section{Data}

Data from the first two experiments were pooled together. The control conditions (long [block 1] and flat) were merged into a new condition named baseline. Block 2 of the long condition in Experiment 1 was renamed transfer, as, due to counterbalancing, participants who completed this condition had previously completed the brief condition in block 1, allowing for potential transfer effects. Unlike in Experiments 1 and 2, which used the proportion of fixations averaged over multiple trials, the analysis in this section uses a binary variable, indicating for each fixation whether it landed on the heterogeneous side of the display or not.

\section{Analysis}

A Bayesian fgeneralized multilevel linear model (family $=$ binomial) was used, following the same general approach as above. Fixation number (2-6), block (1-2) and condition (baseline, brief, reward, transfer) were included in the model as categorical variables, while trial number (1-196) was scaled and centered to have mean 0 and standard deviation 1 and included as a continuous variable. Model comparisons will be made using approximate leave-one-out (loo) cross-validation (Vehtari et al., 2017).

\section{Results}

The results of the model including all four variables and their interactions are shown in Figure 6. From this figure we can see that there is a steady improvement in search efficiency for fixations 5 and 6 over the course of the experiment (trials 1 to 196) Interestingly, this learning does not appear to be present for the initial first few fixations. (As we have noted elsewhere, eventually, all participants spend the majority of their time fixating the heterogeneous side of the display if they have not yet found the target, and so ceiling effects on higher fixation numbers [i.e. $>10$ ] limit the opportunity to learn.)

While it is clear from Figure 6 that fixation number and trial have an effect on the likelihood of a fixation to be directed toward the heterogeneous side of the display, it is less clear whether block has an effect above and beyond what can be accounted for by trial and whether there are any differences between our experimental manipulations. To investigate this, we fit four, simpler versions of the model, each dropping one of our four factors, along with all related interactions.

The model weights are shown in Table 1 . We can see that, in line with our conclusions based on Figure 6, the models with trial and fixation number removed perform poorly. We can also see that the model that ignores block performs poorly, suggesting that a steady improvement from one trial to the next is not enough to account for the rate of learning seen in our participants. The two models that contain block, trial number, and fixation number receive over $80 \%$ of the weight, split approximately equally between the two of them. Given that the model without the condition variable is nested within the full model, this is equivalent to halving the model coefficients for condition and all related

Figure 6

The Proportion of Fixations 2 Through 6, Made to the Heterogeneous Side of the Display for the Four Conditions in Our Experiments

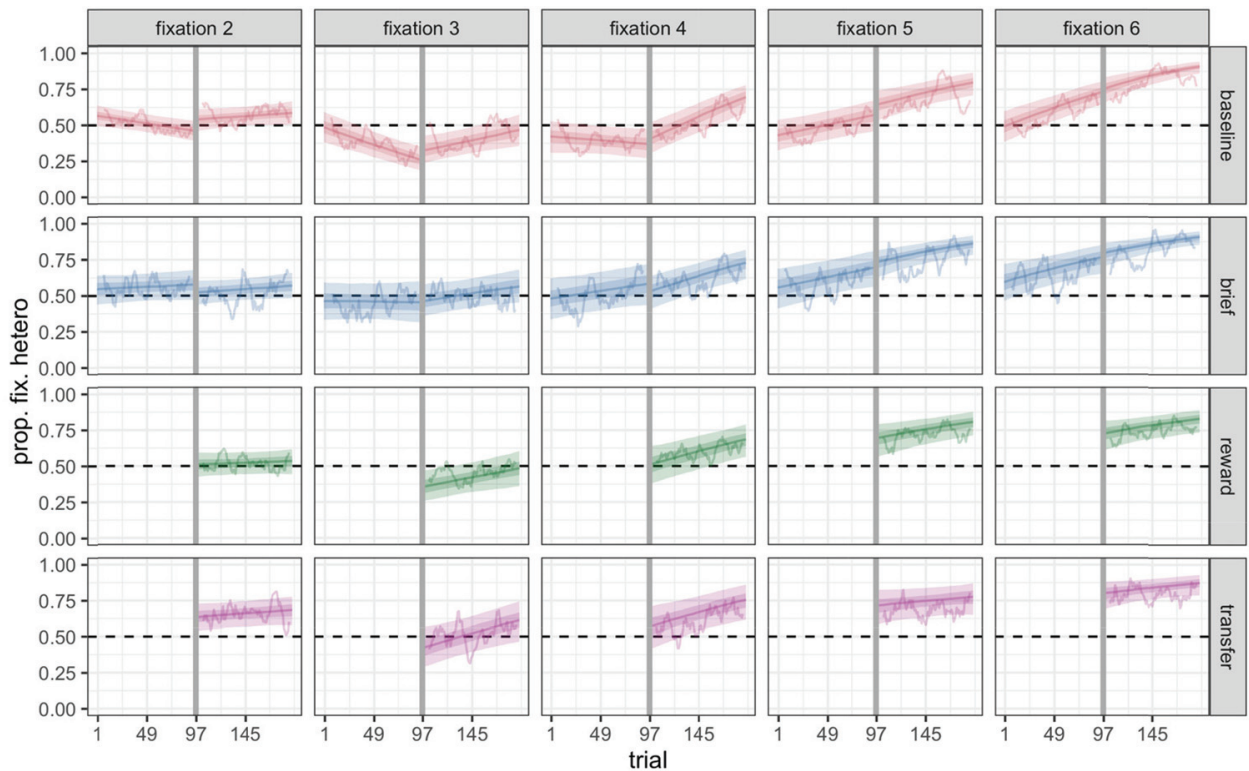

Note. The wiggly line shows a rolling average of the empirical data, while the shaded regions show the 50\% and 90\% HPDI regions for the regression lines. The vertical gray line shows the split between the first and second block. See the online article for the color version of this figure. 
Table 1

Model Stacking Weights

\begin{tabular}{lcc}
\hline \multicolumn{1}{c}{ Model } & $N$ of parameters & Model weights \\
\hline All & 80 & 0.424 \\
Ignore trial & 40 & $<0.001$ \\
Ignore block & 40 & 0.063 \\
Ignore condition & 20 & 0.430 \\
Ignore fixation & 16 & 0.083 \\
\hline
\end{tabular}

Note. These show the weight we should put on each model's prediction to generate the best average prediction.

interactions. As the full model (see Figure 6) already shows little difference between conditions, and in the interest of parsimony (the addition of condition and its interactions requires over 50 new coefficients to be estimated from the data), we conclude that there is little evidence that the introduction of the short time limit, or the financial incentive that we used, had sizable impact on the rate of which participants improved their search efficiency over time.

\section{Saccadic Latency Analysis}

A plausible explanation for inefficient fixation behavior we also considered is that the homogeneous side may appear more visually salient and out-compete the heterogeneous side for control of attention. In this case, a failure to inhibit a reactive saccade to the homogenous side could explain why participants look here, despite the fact that it provides no information. If this is the case, the latency of initial saccades to the homogeneous side should be faster than to the heterogeneous side. To evaluate this, we examined the latency of the first saccade on each trial as a function of where that saccade was directed (homogeneous or heterogeneous side). We excluded one observer who had a median latency greater than $1100 \mathrm{~ms}$ (although the results are essentially the same whether this person is included or not).

The mean of the median latencies showed no difference with the direction of the saccade: Across long, brief, flat and reward conditions, no differences between homogeneous and heterogeneous latencies larger than $5 \mathrm{~ms}$ were observed. A table with the results across conditions is presented in the online supplementary materials. Saccades to the homogeneous side are not executed faster than saccades to the heterogeneous side.

\section{Discussion}

These experiments tested the hypothesis that the suboptimal fixation strategies observed in previous studies arose because participants did not consistently prioritize fast search. That is, we considered the possibility that a persistent tendency to fixate locations that provide no new information could occur because most individuals were not trying to search efficiently. Were this true, stronger incentives to search quickly should have been effective in reducing individual variation, bringing all individuals closer to an efficient strategy. We replicated the previous pattern of wide individual differences in search strategy, and in two experiments, we found that experimentally inducing incentives to search more quickly did not have a substantial effect on fixation strategies. We can therefore rule out insufficient motivation as the explanation for inefficient fixations. Whether the participants were already motivated in previous studies in the first place, or whether increasing motivation just has no bearing on efficiency, we cannot discriminate from these experiments. Still, we saw consistent gradual increases in fixation efficiency with practice, irrespective of whether we put time pressure on participants or rewarded them for faster performance. Exploring these timecourse effects further revealed that the first few fixations in each trial were impervious to practice and that it was the efficiency of the fourth to sixth fixation that gradually improved. These observations provide some preliminary insights into how search strategies evolve over time.

The SHLS task employed in these experiments provides a straightforward metric of fixation efficiency that can be easily compared across conditions. In previous studies, we verified that participants can easily spot the target using peripheral vision when it appears on the homogeneous side, demonstrating that fixations directed to this side are superfluous and can only slow search (Nowakowska et al., 2017). We have also shown that the tendency to fixate the homogeneous side of the search array is nonetheless a common behavior among the majority of individuals and that it persists over multiple testing sessions (Clarke et al., 2020; Nowakowska et al., 2019). In the two experiments reported here, we show a similarly wide range of behaviors in this task, with some participants highly inefficient, some intermediate, and some highly efficient. The existence of individual differences in strategy suggests eye movements are not driven preferentially to locations that produce the most information, as Najemnik and Geisler (2005) would suggest. However, a stochastic model (Clarke et al., 2016) also cannot explain human search because it would suggest both sides of the search array should be equally likely to be fixated. What is required is a model that can account for variation between individuals and over time.

The current results provide some groundwork for this more comprehensive model of visual search by ruling out one set of possible explanations for individual differences: those based on motivation and differences in speed/accuracy trade-offs. Although these manipulations of motivation did not lead to changes in strategy, more efficient strategies did gradually emerge with practice: Participants improved in the second session regardless of the presence of a response deadline or offer of additional incentive. Using a task with a similar rationale, we previously tested the same 20 participants each day for five days and found gradual improvements in strategy and high correlation between performance on each consecutive day (Nowakowska et al., 2019). The current study takes this further by documenting the size and progression of these practice effects in a larger set of data. It is possible that some observers use a model-based search that calculates the cost in terms of time or effort against the expected value (Gershman et al., 2015) and, in the context of the current studies, use their knowledge of their own acuity to calculate the most efficient eye landing position, in other words, using computations like those in Najemnik and Geisler (2005) model. Such a strategy could be contrasted with a more error-prone but inexpensive look-up table mechanism (Gershman et al., 2015) whereby fixations are determined by visual and motor constraints, biases, and low-level properties of the visual scene, similar to the stochastic model (Clarke et al., 2016). Increasingly efficient search behavior could occur through a transition from a look-up table mechanism to a modelbased strategy. Alternatively, the look-up table mechanism could simply become better adapted to the search conditions over time. 
Our results showing a gradual improvement with practice, which is insensitive to manipulations of time pressure or reward, suggest the latter account is more likely.

The practice effects observed in the current experiments are partially consistent with previous findings demonstrating that participants can learn to direct eye movements to relevant stimuli in their environment and adjust eye movement statistics to adapt to scenes, fixating sooner and longer on dynamic and potentially hazardous sources of information (e.g., Jovancevic-Misic \& Hayhoe, 2009; Sullivan et al., 2012). These studies have provided support for a set of promising models of visual exploration during complex and naturalistic tasks like walking and driving, in which eye movements are related to the subtasks of ongoing sequences of actions. Reward is an important component in driving efficient information-gathering in these models (e.g., Hayhoe \& Ballard, 2014), however, and it is inconsistent with these models that the reward in Experiment 2 did not accelerate the learning of effective eye movement strategies. Like in those experiments, the current studies rewarded efficient eye movement behavior intrinsically, by rewarding the faster search times that efficient eye movements would engender. A potentially important distinction between our approach and many others, however, is that "efficient" eye movement behavior in our experiment is not as directly defined by the visual context. That is, in many studies, eye movements gathered rewarding information by targeting specific events, locations, or object classes, such as anticipating the bounce-point of a cricket ball and thereby reaching this source of information in time to process the event (Land \& McLeod, 2000). In our studies, the eye movements accrue evidence for a decision, and some locations are a richer source of information than others. This distinction between better and worse sources of information in the visual environment can clearly be learned over time, as our results suggest, but additional reward does not facilitate this learning or lead participants to adopt more efficient strategies.

Previous research from Paeye et al. (2016) provides converging evidence that effective reinforcement of eye movements requires a contingent mapping between an eye movement and an event. Their participants did not spontaneously learn or capitalize on changes in the probability of the target appearing in a particular quadrant of the search area. In contrast, when instead the contingency between the eye movement and the information was made more direct by having the target appear after a saccade to a particular region, or in a particular direction, participants quickly began repeating saccades of this type at a higher frequency. The conditions of our experiment were more similar to the first manipulation (of the target's likely quadrant) in that there was no direct contingency between the saccade and the presentation of the target. Nonetheless, unlike the probability manipulation, the visual information in our scenes is not uniform, which may have permitted the small but steady improvements we observed over time in our experiments. Our results suggest this less direct learning might occur through small, gradual trial-and-error adjustments in scanning behavior.

The timecourse analysis revealed that the first few fixations on each trial were impervious to the effects of practice and that the improvements over time could be largely attributed to the fourth fixation onward. This is an interesting pattern worthy of confirmation and further exploration because of the potential links with existing literature looking at the timecourse of stimulus-driven versus goal-driven overt attention. Several previous visual search studies have shown that saccades executed soon after the search array appears are driven more by visual salience than those executed later (e.g., Donk \& van Zoest, 2008; Hunt et al., 2007; for reviews see Hunt et al., 2010; van Zoest et al., 2010). Eye movements during extended search provide snapshots of dynamic visual, cognitive, and decision processes as they emerge and develop from the onset of the search array to when the response key is pressed. That early fixations are not influenced by practice is consistent with the characterization of early visual processing as "reflexive" and therefore more rigid and predictable than later stages. However, it is also important to note that the large individual differences observed across individuals apply to early as well as later fixations. By the end of each trial, most fixations are directed to the heterogeneous side of the search array; RT is determined in a large part by how quickly participants are able to start focusing fixations on this side, and this is what seems to improve with practice. An important implication is that whether eye movements during search can be characterized as "ideal" could depend not only on the individual but also on the extent of their experience with the task and on which timepoint of a single trial is being considered.

\section{References}

Anderson, B. A., Laurent, P. A., \& Yantis, S. (2011). Value-driven attentional capture. Proceedings of the National Academy of Sciences of the United States of America, 108(25), 10367-10371. https://doi.org/10 .1073/pnas.1104047108

Anderson, B. A., Laurent, P. A., \& Yantis, S. (2012). Generalization of value-based attentional priority. Visual Cognition, 20(6), 647-658 https://doi.org/10.1080/13506285.2012.679711

Anderson, B. A., \& Yantis, S. (2012). Value-driven attentional and oculomotor capture during goal-directed, unconstrained viewing. Attention, Perception \& Psychophysics, 74(8), 1644-1653. https://doi.org/10.3758/ s13414-012-0348-2

Anderson, B. A., \& Yantis, S. (2013). Persistence of value-driven attentional capture. Journal of Experimental Psychology: Human Perception and Performance, 39(1), 6-9. https://doi.org/10.1037/a0030860

Araujo, Ch., Howler, E., \& Pavel, M. (2001). Eye movements during visual search: the costs of choosing the optimal path. Vision Research, 41, 3613-3625. https://doi.org/10.1016/S0042-6989(01)00196-1

Brainard, D. H. (1997). The psychophysics toolbox. Spatial Vision, 10(4), 433-436. https://doi.org/10.1163/156856897X00357

Bürkner, P. (2017). brms: An R package for Bayesian multilevel models using Stan. Journal of Statistical Software, 80(1), 1-28. https://doi.org/ 10.18637/jss.v080.i01

Chelazzi, L., Perlato, A., Santandrea, E., \& Libera, C. D. (2013). Reward teach visual selective attention. Vision Research, 85, 58-72. https://doi .org/10.1016/j.visres.2012.12.005

Chun, M. M., \& Wolfe, J. M. (1996). Just say no: How are visual searches terminated when there is no target present? Cognitive Psychology, 30(1), 39-78. https://doi.org/10.1006/cogp.1996.0002

Clarke, A. D. F., \& Hunt, A. R. (2016). Failure of intuition when choosing whether to invest in a single goal or split resources between two goals. Psychological Science, 27(1), 64-74. https://doi.org/10.1177/ 0956797615611933

Clarke, A. D. F., Green, P., Chantler, M. J., \& Hunt, A. R. (2016). Human search for a target on a textured background is consistent with a stochastic model. Journal of Vision, 16(7), 4. https://doi.org/10.1167/16.7.4

Clarke, A. D. F., Irons, J. L., James, W., Leber, A. B., \& Hunt, A. R. (2020). Stable individual differences in strategies within, but not 
between, visual search tasks. Quarterly Journal of Experimental Psychology: Human Experimental Psychology. Advance online publication. https://doi.org/10.1177/1747021820929190

Clarke, A. D. F., Nowakowska, A., \& Hunt, A. R. (2019). Seeing beyond salience and guidance: The role of bias and decision in visual search. Vision, 3(3), 46. https://doi.org/10.3390/vision3030046

Cornelissen, F. W., Peters, E. M., \& Palmer, J. (2002). The Eyelink toolbox: Eye tracking with MATLAB and the psychophysics toolbox. Behavior Research Methods, Instruments \& Computers, 34(4), 613-617. https://doi .org/10.3758/BF03195489

Della Libera, C., \& Chelazzi, L. (2009). Learning to attend and to ignore is a matter of gains and losses. Psychological Science, 20(6), 778-784. https://doi.org/10.1111/j.1467-9280.2009.02360.x

Donk, M., \& van Zoest, W. (2008). Effects of salience are short-lived. Psychological Science, 19(7), 733-739. https://doi.org/10.1111/j.1467-9280 .2008.02149.x

Dreher, J. C., \& Tremblay, L. (Eds.). (2009). Handbook of reward and decision making. Academic Press.

Eckstein, M. P. (2011). Visual search: A retrospective. Journal of Vision, 11(5), 14. https://doi.org/10.1167/11.5.14

Edland, A. (1994). Time pressure and the application of decision rules: Choices and judgements among multiattribute alternatives. Scandinavian Journal of Psychology, 35(3), 281-291. https://doi.org/10.1111/j .1467-9450.1994.tb00952.x

Gershman, S., Horvitz, E. J., \& Tenenbaum, J. B. (2015). Computational rationality: A converging paradigm for intelligence in brains, minds, and machines. Science, 349(6245), 273-278. https://doi.org/10.1126/science .aac6076

Haider, H., \& Frensch, P. A. (1999). Eye movement during skill acquisition: More evidence for the information-reduction hypothesis. Journal of Experimental Psychology: Learning, Memory, and Cognition, 25, 172-190.

Hayhoe, M., \& Ballard, D. (2005). Eye movements in natural behavior. Trends in Cognitive Sciences, 9(4), 188-194. https://doi.org/10.1016/j tics.2005.02.009

Hayhoe, M., \& Ballard, D. (2014). Modelling task control of eye movements. Current Biology, 24(13), R622-628. https://doi.org/10.1016/j .cub.2014.05.020

Hayhoe, M., \& Rothkopf, C. A. (2011). Vision in the natural world. Wiley Interdisciplinary Reviews: Cognitive Science, 2, 158-166. https://doi .org/10.1002/wcs. 113

Hout, M. C., Robbins, A., Godwin, H. J., Fitzsimmons, G., \& Scarince, C. (2017). Categorical templates are more useful when features are consistent: Evidence from eye movements during search for societally important vehicles. Attention, Perception \& Psychophysics, 79, 1578-1592.

Hunt, A. R., van Zoest, W., \& Kingstone, A. (2010). Attending to emerging representations: The importance of task context and time of response. In A. K. Nobre, \& J. Coull (Eds.), Attention and time (pp. 3-15). Oxford University Press.

Hunt, A. R., von Muhlenen, A., \& Kingstone, A. (2007). The time course of attentional and oculomotor capture reveals a common cause. Journal of Experimental Psychology: Human Perception and Performance, 33, 271-284. https://doi.org/10.1037/0096-1523.33.2.271

Irons, J. L., \& Leber, A. B. (2016). Choosing attentional control settings in a dynamically changing environment. Attention, Perception \& Psychophysics, 78(7), 2031-2048. https://doi.org/10.3758/s13414-016-1125-4

Irons, J. L., \& Leber, A. B. (2018). Characterizing individual variation in the strategic use of attentional control. Journal of Experimental Psychology: Human Perception and Performance, 44(10), 1637-1654. https:// doi.org/10.1037/xhp0000560

Jóhannesson, Ó. I., Kristjánsson, Á., \& Thornton, I. M. (2017). Are foraging patterns in humans related to working memory and inhibitory control? Are foraging patterns in humans related to working. Japanese Psychological Research, 59, 152-166.
Jovancevic-Misic, J., \& Hayhoe, M. (2009). Adaptive gaze control in natural environments. The Journal of Neuroscience: The Official Journal of the Society for Neuroscience, 29(19), 6234-6238. https://doi.org/10 .1523/JNEUROSCI.5570-08.2009

Kristjánsson, Á., Jóhannesson, Ó. I., \& Thornton, I. M. (2014). Common attentional constraints in visual foraging. PLOS ONE, 9(6), Article e100752. https://doi.org/10.1371/journal.pone.0100752

Land, M. F., \& McLeod, P. (2000). From eye movements to actions: How batsmen hit the ball. Nature Neuroscience, 3, 1340-1345.

Leber, A. B., \& Irons, J. L. (2019). A methodological toolbox for investigating attentional strategy. Current Opinion in Psychology, 29, 274-281. https://doi.org/10.1016/j.copsyc.2019.08.008

Liesefeld, H. R., \& Janczyk, M. (2019). Combining speed and accuracy to control for speed-accuracy trade-offs. Behavior Research Methods, 51(1), 40-60. https://doi.org/10.3758/s13428-018-1076-x

Lonnqvist, B., Elsner, M., Hunt, A. R., \& Clarke, A. D. F. (2020). Modeling individual variation in visual search with reinforcement learning. https://doi.org/10.31234/osf.io/suj28

MacLean, M. H., \& Giesbrecht, B. (2015). Neural evidence reveals the rapid effects of reward history on selective attention. Brain Research, 1606, 86-94. https://doi.org/10.1016/j.brainres.2015.02.016

McElreath, R. (2020). Statistical Rethinking: A bayesian course with examples in $R$ and STAN. CRC Press.

Mormann, M., Navalpakkam, V., Koch, C., \& Rangel, A. (2012). Relative visual saliency differences induce sizable bias in consumer choice. Journal of Consumer Psychology, 22, 1. https://doi.org/10.1016/j.jcps.2011 .10 .002

Morvan, C., \& Maloney, L. T. (2012). Human visual search does not maximize the post-saccadic probability of identifying targets. PLoS Computational Biology, 8(2), e1002342. https://doi.org/10.1371/journal.pcbi .1002342

Najemnik, J., \& Geisler, W. S. (2005). Optimal eye movement strategies in visual search. Nature, 434(7031), 387-391. https://doi.org/10.1038/ nature 03390

Najemnik, J., \& Geisler, W. S. (2008). Eye movement statistics in humans are consistent with an optimal search strategy. Journal of Vision, 8(3), 4. https://doi.org/10.1167/8.3.4

Nakayama, K., \& Martini, P. (2011). Situating visual search. Vision Research, 51(13), 1526-1537. https://doi.org/10.1016/j.visres.2010.09 .003

Navalpakkam, V., Koch, C., Rangel, A., \& Perona, P. (2010). Optimal reward harvesting in complex perceptual environments. Proceedings of the National Academy of Sciences of the United States of America, 107(11), 5232-5237. https://doi.org/10.1073/pnas.0911972107

Nowakowska, A., Clarke, A. D. F., \& Hunt, A. R. (2017). Human visual search behaviour is far from ideal. Proceedings of the Royal Society: Biology, 284, Article 20162767. https://doi.org/10.1098/rspb .2016.2767

Nowakowska, A., Clarke, A. D. F., Sahraie, A., \& Hunt, A. R. (2019). Practice-related changes in eye movement strategy in healthy adults with simulated hemianopia. Neuropsychologia, 128, 232-240. https:// doi.org/10.1016/j.neuropsychologia.2018.01.020

Nowakowska, A., Clarke, A. D. F., Sahraie, A., \& Hunt, A. R. (2016). Inefficient search strategies in simulated hemianopia. Journal of Experimental Psychology: Human Perception and Performance, 42(11), 1858-1872. https://doi.org/10.1037/xhp0000250

Paeye, C., Schutz, A. C., \& Gegenfurtner, K. R. (2016). Visual reinforcement shapes eye movements in visual search. Journal of Vision, 16(10), 15. https://doi.org/10.1167/16.10.15

Pelli, D. G. (1997). The VideoToolbox software for visual psychophysics: Transforming numbers into movies. Spatial Vision, 10(4), 437-442. https://doi.org/10.1163/156856897X00366

Pietersa, R., \& Warlopb, L. (1999). Visual attention during brand choice: The impact of time pressure and task motivation. International Journal 
of Research in Marketing, 16(1), 1-16. https://doi.org/10.1016/S0167 -8116(98)00022-6

Rajsci, J., Taylor, J. E. T., \& Pratt, J. (2017). Out of sight, out of mind: Matching bias underlies confirmatory visual search. Attention, Perception, \& Psychophysics, 79, 498-507.

Rajsic, J., Wilson, D. E., \& Pratt, J. (2015). Confirmation bias in visual search. Journal of Experimental Psychology: Human Perception and Performance, 41(5), 1353-1364. https://doi.org/10.1037/ xhp0000090

Small, D. M., Gitelman, D., Simmons, K., Bloise, S. M., Parrish, T., \& Mesulam, M. (2005). Monetary incentives enhance processing in brain regions mediating top down control of attention. Cerebral Cortex, 15(12), 1855-1865. https://doi.org/10.1093/cercor/bhi063

Sullivan, B. T., Johnson, L., Rothkopf, C. A., Ballard, D., \& Hayhoe, M. (2012). The role of uncertainty and reward on eye movements in a virtual driving task. Journal of Vision, 12(13), 19. https://doi.org/10.1167/ 12.13.19

Svenson, O., \& Edland, A. (1987). Change of preferences under time pressure. Scandinavian Journal of Psychology, 28(4), 322-320. https://doi .org/10.1111/j.1467-9450.1987.tb00769.x

Tagu, J., \& Kristjánsson, A. (2020). Dynamics of attentional and oculomotor orienting in visual foraging tasks. Quarterly Journal of Experimental Psychology: Human Experimental Psychology. Advance online publication. https://doi.org/10.1177/1747021820919351

Tatler, B. W., Hayhoe, M. M., Land, M. F., \& Ballard, D. H. (2011). Eye guidance in natural vision: Reinterpreting salience. Journal of Vision, 11(5), 5. https://doi.org/10.1167/11.5.5

Thornton, I. M., de'Sperati, C., \& Kristjánsson, A. (2019). The influence of selection modality, display dynamics and error feedback on patterns of human foraging. Visual Cognition, 27(5-8), 626-648. https://doi.org/ $10.1080 / 13506285.2019 .165800$
Townsend, J. T., \& Ashby, E. G. (1983). Stochastic modeling of elementary psychological processes. Cambridge University Press.

Treisman, A. M., \& Gelade, G. (1980). A feature-integration theory of attention. Cognitive Psychology, 12(1), 97-136. https://doi.org/10.1016/ 0010-0285(80)90005-5

van Herpen, E., \& Trijp, H. C. M. (2011). Front-of-pack nutrition labels. Their effect on attention and choices when consumers have varying goals and time constraints. Appetite, 57(1), 148-160. https://doi.org/10 .1016/j.appet.2011.04.011

van Zoest, W., Hunt, A. R., \& Kingstone, A. (2010). Representations in visual cognition: It's about time. Current Directions in Psychological Science, 19(2), 116-120. https://doi.org/10.1177/0963721410363895

Vehtari, A., Gelman, A., \& Gabry, J. (2017). Practical Bayesian model evaluation using leave-one-out cross-validation and WAIC. Statistics and Computing, 27(5), 1413-1432. https://doi.org/10.1007/s11222-016-9696-4

Wolfe, J. (1998). What can 1 million trials tell us about visual search? Psychological Science, 9(1), 33-39. https://doi.org/10.1111/1467-9280.00006

Wolfe, J. M. (1994). Guided Search 2.0: A revised model of visual search. Psychonomic Bulletin \& Review, 1(2), 202-238. https://doi.org/10.3758/ BF03200774

Wolfe, J. M., \& Van Wert, M. J. (2010). Varying target prevalence reveals two dissociable decision criteria in visual search. Current Biology, 20(2), 121-124. https://doi.org/10.1016/j.cub.2009.11.066

Zhang, J., Gong, X., Fougnie, D., \& Wolfe, J. M. (2017). How humans react to changing rewards during visual foraging. Attention, Perception \& Psychophysics, 79(8), 2299-2309. https://doi.org/10.3758/s13414-017-1411-9

Received July 29, 2020

Revision received December 18, 2020

Accepted January 28, 2021 